\title{
Possibility of Use of Noise Analysis for Identification of Reactor Conditions during Accidents
}

\author{
Tamas Janos Katona \\ MVM Nuclear Power Plant Paks Ltd., Paks, Hungary \\ Email: bata01@t-online.hu
}

Received April 4, 2013; revised May 3, 2013; accepted May 14, 2013

Copyright (C) 2013 Tamas Janos Katona. This is an open access article distributed under the Creative Commons Attribution License, which permits unrestricted use, distribution, and reproduction in any medium, provided the original work is properly cited.

\begin{abstract}
Knowledge and control of the thermo-hydraulic conditions in the reactor is required for the effective accident management. Dedicated and qualified for harsh environment instrumentation has to be in place for this purpose. Experience of the Fukushima Dai-ichi plant and the lessons learned from the European stress tests demonstrated that alternative and divers tools and methods are needed for the identification of reactor condition in extreme situations. In the paper the feasibility of development of an alternative accident monitoring via well-known noise diagnostics methods is proposed and demonstrated. The possibility of identification of reactor accident conditions using temperature and pressure fluctuations, noise of the neutron and gamma field is considered on the basis of research achievements in reactor noise. As an example the use of pressure fluctuations for accident monitoring is presented.
\end{abstract}

Keywords: Accident Management; Nuclear Power Plant; Post-Event Condition; Noise Diagnostics; Pressure Fluctuations

\section{Introduction}

One of the most complex cases for assessing the nuclear power plant safety is the evaluation of the response of the plant to earthquakes: whether the reactor can be shut down, cooled-down, the residual heat can be removed from the core, and the radioactive releases can be limited below the acceptable level. As per design, set of plant systems, structures and components (SSCs) are in place and shall remain functional during and after the earthquake for ensuring these basic safety functions. Experiences show that plants can survive much larger earthquakes than those considered in the design base, as it was the case of Kashiwazaki-Kariwa NPP, where the safety classified structures, systems and components survived the Niigata-Chuetsu-Oki earthquake in 2007 without damage and loss of function [1]. Similarly, thirteen nuclear units in the impacted area on the East-shore of the Honshu Island demonstrated high earthquake resistance 11th of March 2011 [2].

In spite of high designed-in capacity, in case of a beyond design base earthquake or an unfortunate combination of external and/or internal events, these SSCs can be damaged and lost. In this case a minimum configuration for emergency heat removal to the ultimate heat sink and minimum power supply has to be arranged as emergency measures including minimum instrumentation configuration for the control of reactor condition.

The practical questions to be answered for the operators after the event are as follows:

- In case of relative small earthquake it has to be known, whether the operation can be continued safely. This is the issue of the Kashiwazaki-Kariwa plant.

- In case of large earthquake, the damaged condition of the plant has to be immediately assessed for the emergency management reasons. This is the Fukushima NPP case.

For the proper knowledge of the situation at the plant after the event, both the assessment of the structural integrity and functionality and identification of the thermo-hydraulic conditions in the reactor and primary circuit, etc. are required. The case of Kashiwazaki-Kariwa plant initiated the research activity for development of methodologies for quick assessment of integrity of SSCs (see e.g. [3]). The experience learned at Fukushima Dai-ichi reactors shows that the identification of the reactor thermo-hydraulic condition is unavoidable for the definition and implementation of the accident management measures. The Fukushima-Dai-ichi case demonstrated the difficulties caused for example by loss of information on the reactor water level [4]. The knowledge 
of the thermo-hydraulic condition in the containment, spent fuel pool and in components involved or affecting the implementation of measures is also important.

Obviously, in case of severe accident, application of any methods for the control of the reactor/plant condition can be justified, when the normal and accident instrumentation do not provide enough information or even fail. As it shown in the Fukushima Dai-ichi case the tasks of identification of the accident conditions in the reactor is far more complex than the known BWR water level measurement issue [5-7].

The achievements of the theoretical and experimental noise investigations can be used for the development of a monitoring of accident conditions in the reactor. Use of the noise analysis methods can provide additional information to those obtained by conventional methods from the signals of the accident instrumentation. This is a possible defense-in-depth level four application of noise diagnostics.

In the paper the possibility of the application of different noise analysis methods for identification of accident conditions is proposed and discussed. This is a novel idea since the noise diagnostic methods were intended to use for early identification of deviations from normal reactor conditions. The idea of post-accident diagnostics is based on the phenomenology of thermo-hydraulic processes going on in the reactor and primary system during the accident sequence. The correlation between fluctuations of reactor parameters and post-accident conditions is qualitatively demonstrated for fluctuations of coolant density, temperature and pressure in the reactor. The feasibility of identification of different reactor conditions via measurement and analysis of pressure fluctuation is shown via calculation of hydro-acoustic resonances for simplified models of the reactor and primary system. Different cases have been analyzed, such as: water in reactor and primary loops, steam-water mixture in the reactor and water in loops and steam in the reactor and in the loops. It is also shown that the hydro-acoustic resonances of the sensing lines do not inhibit the proper interpretation of measured pressure fluctuations with regard to the conditions in the reactor.

The demonstration of applicability of noise analysis methods for identification of post-accident conditions is based on the available research results. The references in this paper are representative, but a full-scope review of the noise diagnostics results applicable for accident monitoring exceeds the frame of recent paper. Moreover, majority of the referred papers are from eighties and nineties, when the basics of reactor noise theory were developed and the fundamental experiments performed. These results turn out to be absolutely state-of-the-art in context with the new application for accident monitoring. The main theoretical and practical issues that require further research effort are also indicated in the paper.

\section{Idea of Severe Accident Noise Diagnostics}

The basic idea proposed in recent paper is rather trivial: the fluctuations of the thermo-hydraulic parameters provide information regarding thermo-hydraulic condition additional to the information obtained from the average or filtered values of the same parameters. The safety relevant variables defining the conditions in the reactor during the accident process are the characteristics of the water-steam-gas mixture cooling the core, i.e.

- composition (density, i.e. the steam and/or gas content in the water),

- thermo-hydraulic properties (temperature, pressure, flow-rate),

- spatial distribution of the phases (flow-pattern and water level in the reactor).

The thermo-hydraulic parameters are enormously varying in time and space in the core as well as in the primary system during the accident. The two-phase flow is cooling the core at the very beginning of the sequence, afterwards, up-to the complete dry-out, a stagnant twophase water-steam or steam non-condensable gas mixture fills the reactor pressure vessel and finally, after successful rewetting and refilling of the core, a stagnant or flowing borated water-steam mixture cools the core. It is obvious, that the fluctuations of state parameters of the medium in the reactor and primary system are as informative as the filtered values.

Some of the safety relevant parameters and thermohydraulic state variables are directly measurable like temperature and pressure. However, the time averaged values of these parameters are not selective with respect to the phases of the cooling mixture (water, steam or gas). Moreover, the composition and time-space distribution of the cooling mixture and the flow-rate can't be measured directly. As it shown below, the noise analysis methods can provide these directly not measurable data.

Accident monitoring can be built on the measurement and analysis of the noise of state variables of the medium, and fluctuation of other reactor parameters if

- the reactor conditions during the accident process can be correlated to and identified by the stochastic features of fluctuations of state parameters, and

- the instrumentation or part of the instrumentation is available and operable for the measurement of the fluctuations of the state parameters during the accident process.

The research results and application experiences of the reactor noise diagnostics provide rather high certainty for the positive outcome of the application of noise methods for the identification of severe accident conditions in the reactor/plant.

The development of reactor noise diagnostics has been 
motivated by the need of early identification of deviations form the normal operating conditions. With this respect, the reactor noise diagnostics is part of the defense-in-depth level-one technical features. The noise diagnostic tools and techniques are implemented at the nuclear power plants in different extents; some plants use the noise diagnostics rather limited, e.g. the loose part monitoring [8] or sensor-diagnostics [9], some other plants, e.g. the Paks NPP, operate complex reactor noise diagnostic systems [10]. The noise diagnostic methods can also be applied for specific reactor-physics purposes [11]. There were some attempts to apply the noise diagnostic methods for controlling the reactor conditions rather far from normal operation [12]. Essential progress has been made in the area of identification of thermohydraulic anomalies via noise diagnostics. Most important achievements of these researches, applicable for the accident process diagnostics will be referred below.

The measurement of noise of the reactor parameters of interest can be performed either using the accident instrumentation or by specific, low-inertia detectors and transmitting lines qualified for harsh environment. The possibility of installation of detectors and transducers appropriate for noise measurement is demonstrated by experience of several plants operating reactor noise diagnostics systems. The resolution of the stress test issues will obviously require the implementation of minimum instrumentation configuration for identification and control of the plant condition during the accident sequence. This instrumentation can also be used for the noise measurement, if the sensors are properly selected.

\section{Diagnostics Based on the Fluctuation of the Coolant Density}

During the accident sequence the density fluctuations of the cooling medium (including variation of the reactor level) are in place even if the medium is stagnant and the reactor shutdown. The neutron and gamma field in and around the reactor is modulated by the time and space distribution of the density of the medium. There is a strong correlation between the neutron and gamma field fluctuations and fluctuations of time-space distribution of the density/level of the medium in the reactor. This phenomenon is used for condition diagnostics and measurement of flow velocity via measurement and analysis of in-core and/or out of vessel neutron noise. Using neutron noise the flow pattern can also be identified. A selection of relevant papers are given in references, see e.g. [13-22]. Use of neutron and/or gamma telescopes for detection of void fraction and water/gas volumes in the reactor has been proposed already late seventies [23]. There are also results showing the possibility of the detection of the phase distribution (density and water level) of the coolant based on the gamma radiation distribution outside the reactor pressure vessel. In [24] the measured ex-vessel $\gamma$-signals are input values of a neural network, so that the water levels can also be determined in nonstationary processes.

\section{Use of Temperature Signals for Condition Diagnostics}

The use of core exit temperature signals for accident management is already recognized. An extensive overview of experimental and analytical results for core exit temperature signals is given in [25].

The temperature sensors are rather robust, able to withstand relative large deviations from the normal operating conditions. The usual way of the installation of the temperature sensors (for example in protecting tubes) restrains the measurement of the noise component of the signal. Nevertheless, certain possibility exists for the low-frequency noise measurements with normal industrial thermocouples and thermometers.

Features of the temperature noise measured at the core exit correlated with the characteristics of the two-phase flow including phase distribution and flow-velocity (see e.g. $[26,27])$. The noise of the thermocouples or resistance thermometers in the loops can also be used for the diagnostics of the flow in the primary loop.

\section{Diagnostics Based on the Pressure Noise Signals}

\subsection{General Considerations}

The hydro-acoustic features (hydro-acoustic resonances, parameters of the standing waves) in the reactor and primary system depend on the thermo-hydraulic conditions in place, i.e. on the density, volume and compressibility (speed of sound) of the water/steam/gas mixture in the system. The speed of sound is extremely sensitive to the void content in liquid: some per cents of steam or gas in water result in one or two order of magnitude degreasing of the sound speed. Generally, the speed of sound multicomponent, multi-phase medium in pipe or channel depends also on the flow-pattern, i.e. whether the dispersed phase is in form of small bubbles or large slugs. Basic in formation on the sonic velocity in two-phase medium can be found e.g. in [28-31].

The hydro-acoustic resonances in the reactor and primary system have been intensively studied early eighties mainly in context with vibration diagnostics of the in vessel structures, piping and steam-generator heat exchanging tubes. Theoretical and experimental investigations and hydro-acoustic modeling of the reactor and primary system have been published, e.g. for the VVERtype reactors; see [32-41]. Rather intensive research and development activity is still going on in this area for example in Russia, see e.g. $[42,43]$. 
Theoretical description of hydro-acoustic fluctuations in a distribution systems consisting of pipes and volumes will be presented here very limited, focusing only on the simple cases analyzed. For the sake of simplicity the damping of the waves will be neglected. Thus, the pressure and velocity fluctuations, $\tilde{p}(l)$ and $\tilde{v}(l)$ at the end of a pipe 1, where 1 is the length of the pipe, can be calculated using Equation (1), if the pressure and velocity fluctuations, $\tilde{p}(0)$ and $\tilde{v}(0)$ at the other end of the pipe segment at $x=0$ are known:

$$
\begin{aligned}
& \tilde{p}(x)=\tilde{p}(0) \cos \left(\frac{\omega}{c} x\right)+i \rho c \tilde{v}(0) \sin \left(\frac{\omega}{c} x\right) \\
& \tilde{v}(x)=\frac{i}{\rho c} \tilde{p}(0) \cos \left(\frac{\omega}{c} x\right)+\tilde{v}(0) \sin \left(\frac{\omega}{c} x\right)
\end{aligned} .
$$

Equation (1) can be written in matrix form:

$$
\left|\begin{array}{l}
\tilde{p}(x) \\
\tilde{v}(x)
\end{array}\right|=\left|\begin{array}{cc}
\cos \left(\frac{\omega}{c} x\right) & i \rho c \sin \left(\frac{\omega}{c} x\right) \\
\frac{i}{\rho c} \sin \left(\frac{\omega}{c} x\right) & \cos \left(\frac{\omega}{c} x\right)
\end{array}\right|\left|\begin{array}{l}
\tilde{p}(0) \\
\tilde{v}(0)
\end{array}\right| .
$$

Equation (2) can be written in generic form as follows:

$$
\underline{X}_{k-1}=\underline{\underline{M}}_{k-1, k} \underline{X}_{k} \text {. }
$$

For the closed system like the primary system with the reactor, the condition $\underline{X}_{k}=\underline{X}_{0}$ is valid. Consequently the system can be described as follows:

$$
(\underline{\underline{M}}-\underline{\underline{E}}) \underline{X}_{k}=0
$$

and the resonant frequencies are defied by the equation:

$$
\operatorname{det}(\underline{\underline{M}}-\underline{\underline{E}})=0 \text {. }
$$

Introducing the acoustic impedance $Z=\tilde{p} / \tilde{v}$, analysis of the distribution system consisting of pipes and volumes can be performed utilizing the analogy with electrical circuits. Thus the Equation (1) can be rewritten in the form:

$$
Z_{0}=\frac{Z_{l}+i \cdot \rho \cdot c \cdot \operatorname{tg}\left(\frac{\omega}{c} l\right)}{1+Z_{l}+\frac{i}{\rho \cdot c} \cdot \operatorname{tg}\left(\frac{\omega}{c} l\right)} .
$$

For example, the impedance of a volume $V$ is:

$$
Z_{V}=\frac{\rho \cdot c^{2}}{i \cdot \omega \cdot V}
$$

Condition for resonance is if the impedance of the system is equal to zero.

\subsection{Conditions for Feasibility}

The following questions have to be answered while considering the possibility of accident monitoring via meas- urement and analysis of pressure fluctuations:

- Whether the detection of the pressure fluctuations is possible.

- Whether the changes of conditions in the reactor and primary circuit during accident sequence will result in significant changes of the hydro-acoustic behavior of the system, which result in convincing changes of the features of the pressure fluctuations.

The measurability of the pressure fluctuations will be discussed in Section 5.3.

The correlation between hydro-acoustic resonances and reactor conditions is shown in Section 5.4.

\subsection{Behaviour of the Sensing Lines}

From practical point of view the aspect of measurability of the pressure fluctuations in the reactor seems to be decisive. The pressure fluctuations can be detected, if

- the pressure transducers itself are of low-inertia, i.e. they are able to detect the low-frequency pressure fluctuations,

- the detectors can withstand the accidental conditions, and

- the changes of the resonant frequencies of the sensing line itself will not inhibit the identification of the changes of the pressure fluctuations in the reactor.

It seems to be manageable to install low-inertia pressure transducers that can survive accidental conditions far from the reactor, mounted at the end of a long sensing line. The pressure sensor and sensing line issue is analyzed in [9].

In this paper the third condition of feasibility will be analyzed.

For the analysis of detectability a simple model of the sensing line, closed-up at one end by the detector/ transducer and on the other end by the reactor has to be analyzed, see Figure 1.

Sensing line resonance-frequencies have been defined for the combinations of conditions in the line and in the reactor and primary system as follows:

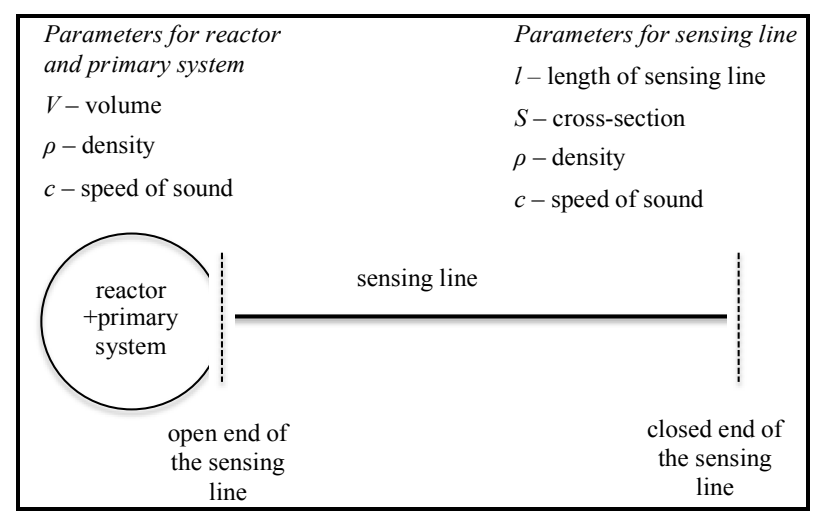

Figure 1. Model of the sensing line. 
- three different condition of the medium in the line: 1) water; 2) two-phase with low void content; 3) steam.

- three different conditions in the reactor and primary system: 1) water; 2) two-phase with low void content; 3) steam.

Calculation of the resonance frequencies the impedance formulation has been used (see Equations (6) and (7)).

The length $l$ of the sensing line and cross-sectional area, $\mathrm{S}$ are set equal to typical plant line dimensions, particularly the length $l=50 \mathrm{~m}$ and the total volume of the reactor and primary system $V=200 \mathrm{~m}^{3}$.

In the calculations some typical values for the coolant densities, $\rho$ and of speed of sound, $c$ are selected, corresponding to the conditions in the reactor and the loops as well as in the sensing line. The primary system and the sensing line can be filled either with water, 2-phase water-steam mixture (low void) or steam.

Results of the calculation of the sensing line impedance are plotted in Figure 2 for all combinations of the typical conditions in the sensing line and in the reactor as it is shown in the Table 1. Condition for the resonance is if the impedance is equal to zero. In the Table 1 the values of the first resonance-frequencies are given.

As it is shown from the Table $\mathbf{1}$ and Figure 2 the hydro-acoustic behavior of the sensing line is depending very much on the value of the sonic velocity, which is very much depending on the vapor/gas content of the medium in the sensing line. The values of the resonances are slightly influenced by the density of the medium.

From the point of view of feasibility of diagnosis most important result is that the sensing line resonances are practically independent from the total impedance of the primary system, except of cases when the volume is set very low (if $V$ is much less than $1 \mathrm{~m}^{3}$ ).

\subsection{Correlation between Reactor Condition and Hydro-Acoustic Features of the System}

Several studies exist for the calculation of hydro-acoustic resonance frequencies in VVERs (see [32-41]). The models include usually the reactor considered as a volume, piping of one loop with steam-generator and the pressurizer as it shown in upper part of the Figure 3. For the demonstration of the feasibility of accident monitoring a very simple model of the primary system will be used, see in the lower part of Figure 3.

Typical for a pressurized-water reactor length of the primary loop 1 and the cross-section $\mathrm{S}$ are selected. The length is equal to $50 \mathrm{~m}$, and the volume of the reactor is set for $50 \mathrm{~m}^{3}$. Typical values for the coolant densities, $\rho$ and of speed of sound, c are selected, corresponding to the cases when the reactor and the loops are filled either with water, 2-phase water-steam mixture (low void) or steam.

Calculation of the resonance frequencies the matrix formulation has been used (see Equations (1) to (5)).

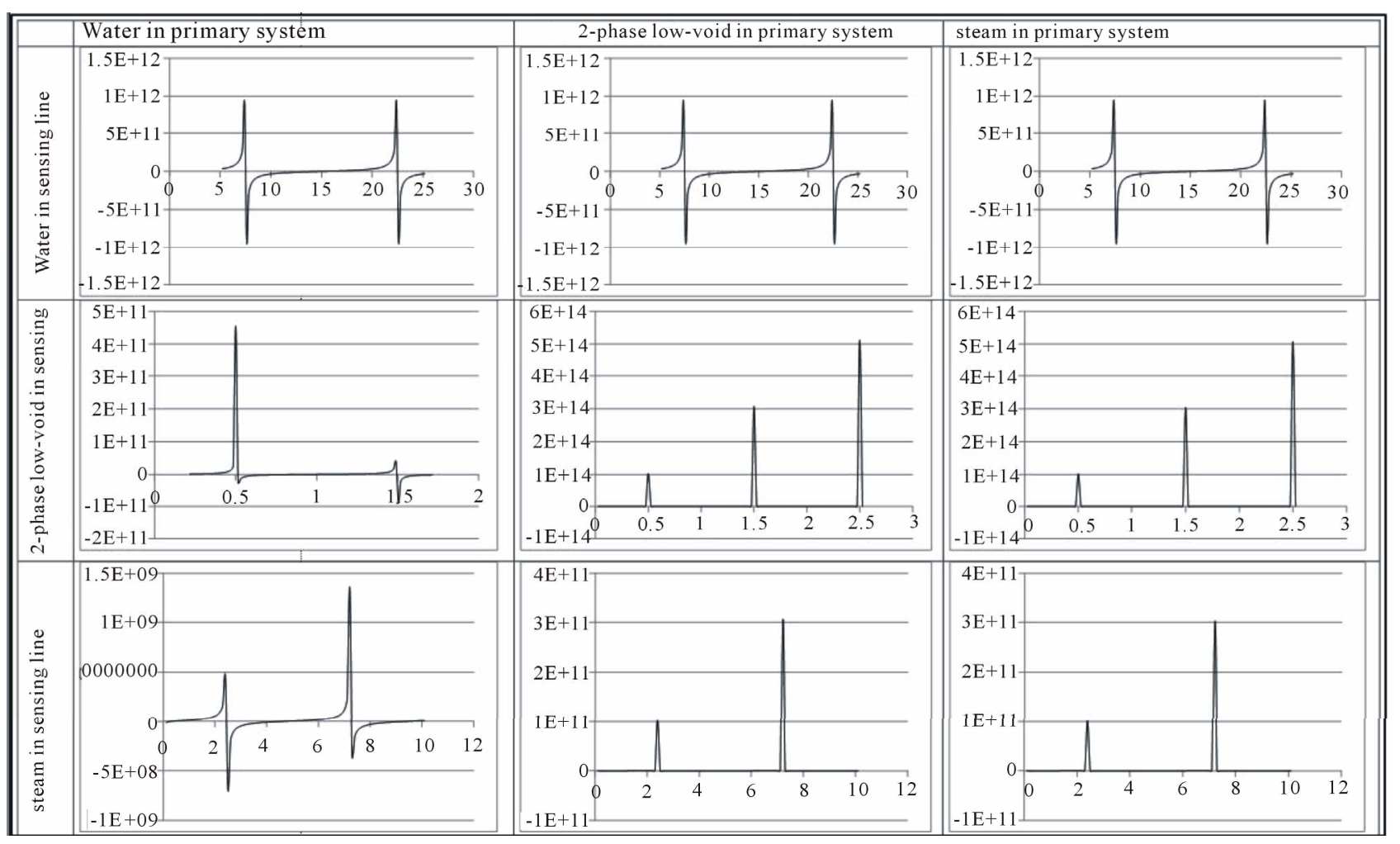

Figure 2. Impedance of the primary system and the sensing line. 
Table 1. Calculated first resonance-frequencies of the sensing line (Hz).

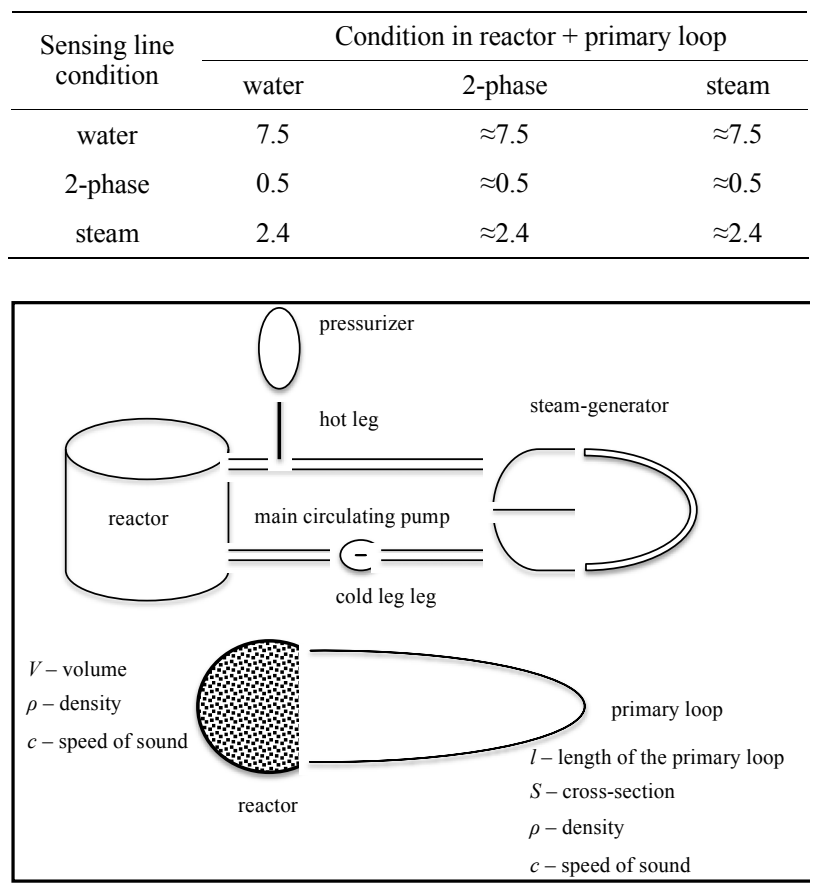

Figure 3. Model of the reactor + primary loops.

The first resonance-frequencies for all combinations of the typical conditions in the reactor and primary loop are given in the Table 2 .

Results of the calculation of the system resonances are plotted in Figure 4 for the selected cases.

Analyzing the results shown in Figure 4, it is obvious that in this simple model the resonance-frequencies are related to the loop. However, those frequencies depend on the boundary conditions at the ends of the primary pipe, i.e. on the condition in the reactor. That is shown in the Figure 5, where the loop is filled with steam and the conditions in the reactor vary. Obviously, the high-pressure case in the loop can also be differentiated from the low-pressure one.

\subsection{Limitations of the Calculations}

Thus, the pressure fluctuations in the primary system (reactor and loops) can be detected by pressure transducers mounted on long sensing lines, if the properties of the medium would be known. Hence it is not the case under accident conditions, calculations have to be made in advance for all feasible cases for the sensing line hydroacoustic behavior. If the sensing line resonances are known, they can be identified and excluded from the evaluation of the condition of the primary system.

In this model, the case with low-void two-phase medium in the reactor can't be separated. However this is the limitation of the simple model used and not the limi-
Table 2. Calculated first resonance-frequencies of the system reactor + primary loop $(\mathrm{Hz})$.

\begin{tabular}{cccc}
\hline \multirow{2}{*}{$\begin{array}{c}\text { Condition in } \\
\text { loops }\end{array}$} & water & 2-phase & steam \\
\cline { 2 - 4 } water & $\approx 30$ & $\approx 7.5$ & $\approx 7.5$ \\
2-phase & $\approx 2$ & $\approx 2$ & $\approx 2$ \\
steam & $\approx 12$ & $\approx 12$ & $\approx 12$ \\
\hline
\end{tabular}

tation of the method in general.

The rather simple calculations above show that the most resonance frequencies are very sensitive to the sound velocity. Since the hydro-acoustic resonances are most sensitive to the sound velocity, the calculated resonances can be fitted to the measured one practically via tuning the value of sound velocity. Thus, the identification of the thermo-hydraulic conditions in the system would lead to correlation of the adjusted sound velocity to the thermo-hydraulic conditions characterized by that value of sound velocity. Consequently, the most serious hindrance for the feasibility of pressure noise diagnostics of post-accident condition might be the lack of knowledge of sound velocity in non-equilibrium two-phase medium in the reactor and sensing line.

\section{Tasks for the Further Research}

\subsection{Basic Research Areas}

For the development of the accident monitoring via noise analysis two basic research areas can be identified:

- investigation of the correlation between the accident conditions and fluctuation of state variables;

- investigation of the conditions, tools and techniques for measurement of noise signals during accident situation.

The accident processes are widely studied analytically and empirically. The state variables show stochastic character practically in all phases of the accident scenarios. Though that phenomenon is well known and observed in the tests, the fluctuating part of the variables is generally neglected in the deterministic description of the scenarios. First task for the future development of the noise analysis methods for accident monitoring have to be the identification of stochastic features of the state variables for each phases of the accident scenarios (one phase heat removal, two-phase heat removal, dry-out, rewetting and refilling, etc.) via reanalysis of existing experimental information and results of deterministic calculations. Identifying the possible phases of the process, the stochastic features of the fluctuation of state variables for each phase/condition can be mapped in advance. Focused experiments might be practicable for the justification of the criteria (features of the fluctuations) for the identification of particular conditions in the reac- 


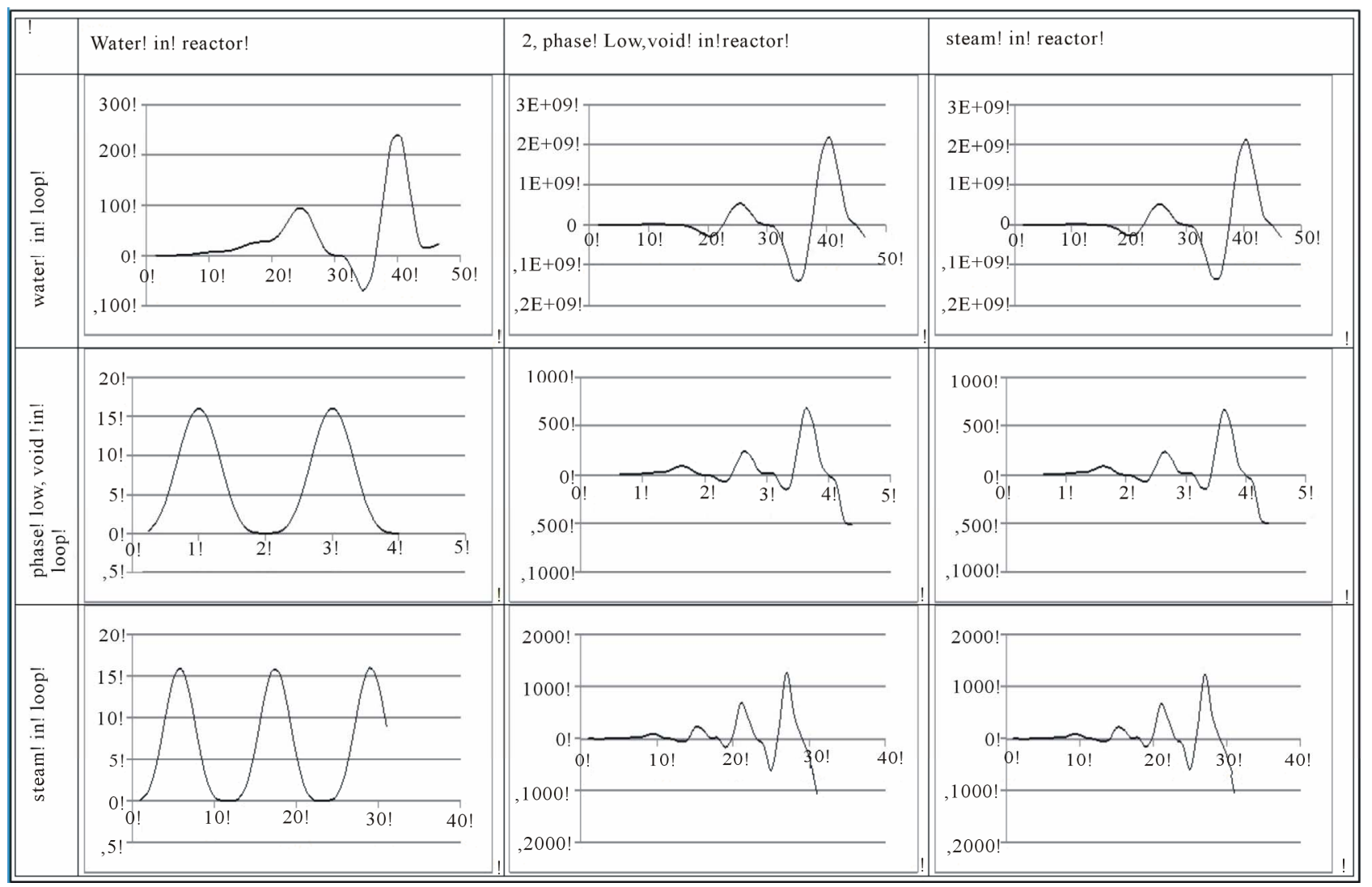

Figure 4. Resonances for the reactor and primary system.

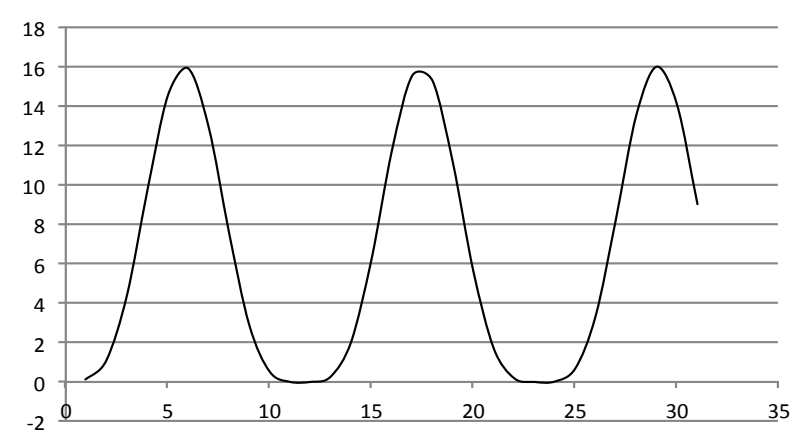

(a)

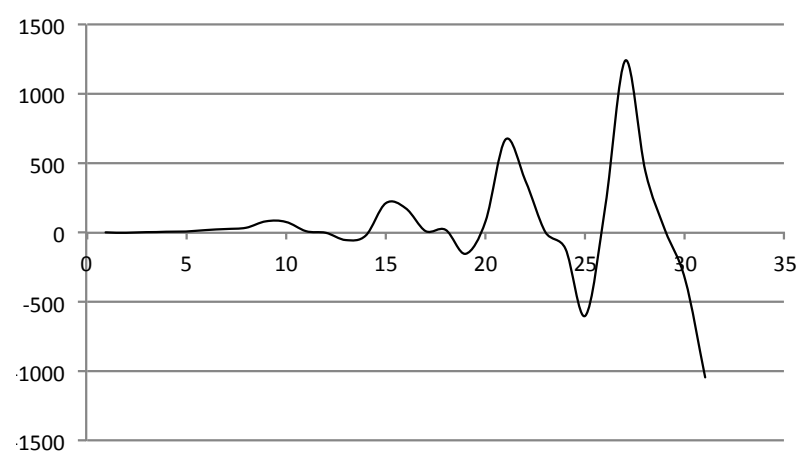

(c)

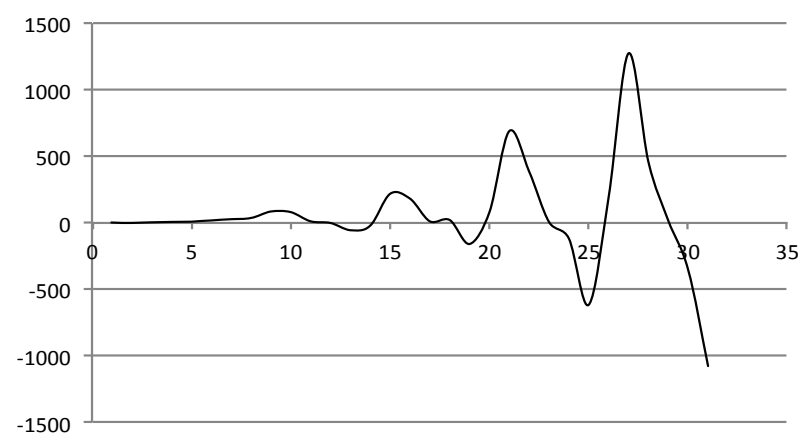

(b)

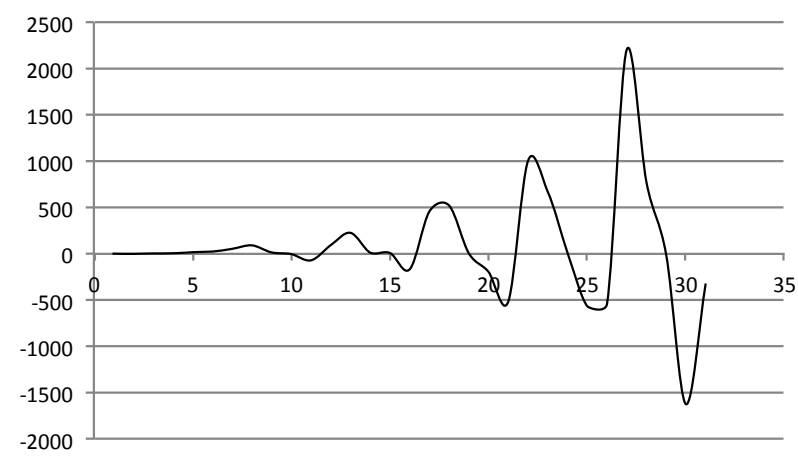

(d)

Figure 5. Higher harmonics in the system with varying reactor condition and steam in the primary loop. (a): Water in the reactor; (b): Two-phase, low-void in the reactor; (c): Steam in the reactor; (d): Steam in the reactor-low pressure. 
tor. The features of the fluctuation of the gamma and neutron field due to fluctuations of the density of medium in the reactor have to be investigated, too.

The need of the investigation of the feasibility of the measurement of noise signals under accident condition (instrumentation, data acquisition and analysis) does not require further explanations.

\subsection{Research Tasks for Pressure Noise Diagnostics}

Regarding use pressure fluctuations for the identification of accident condition in the reactor following tasks for further research can be identified besides the mentioned above issues:

- Complex hydro-acoustic models have to be developed for each typical situation of the accident sequence. It seems to be reasonable to develop detailed reactor models, consisting of several volumes and connections between them. A tree-dimensional modeling of standing waves in the reactor pressure vessel can also be considered.

- Description of pressure waves in two-phase (two-or three component) non-equilibrium medium requires essential effort. The issue has been investigated mainly in relation to the loss-of-coolant accidents (see e.g. [28-31]). Generally, the sound velocity depend on the steam/gas phase content (void) and distribution of the phase (bubble, slug-plug, spray, etc.), on the interphase mass, momentum and heat transfer and on the frequency. Obviously, reasonable assumptions have to be made with regard to the sound velocity on the basis of rigorous analyses.

- In non-equilibrium two-phase medium pressure noise can be generated e.g. by collapsing steam bubbles. This type of phenomenon can also be used for the identification of the condition in the core (see e.g. [44]).

\section{Conclusion}

In case of accidents, when the information for the identification of the condition in the reactor can be very limited due to damages and function losses of the measuring system, use of all possible methods can be justified for the support of accident management. Measuring the noise of the available signals and application of the noise analysis methods can enhance the information from the available measurements. As it is demonstrated for the feasibility of using pressure fluctuations for accident monitoring, the existing knowledge and experience in the field of noise diagnostics and related research provide solid basis for the future research regarding application of noise analysis for identification of accident condition in the reactor.

\section{REFERENCES}

[1] International Atomic Energy Agency, "Preliminary Findings and Lessons Learned from the 16 July 2007 earthquake at Kashiwazaki-Kariwa NPP," Mission Report, Vienna, 2007.

[2] IAEA, "International Fact Finding Expert Mission of the Nuclear Accident Following the Great East Japan Earthquake and Tsunami," 2011.

http://www.iaea.org/newscenter/news/2011/japanmission. html

[3] T. J. Katona, "Possibility for Using Damage Indicators for Definition of Nuclear Power Plant Seismic Fragility," Proceedings of the 8th International Conference on Structural Dynamics, Leuven, 2-4 July 2011, pp. 234-237.

[4] Japanese Government, "Report of Japanese Government to IAEA Ministerial Conference on Nuclear Safety-Accident at TEPCO's Fukushima Nuclear Power Stations," 7 June 2011, pp. 44-49.

http://www.iaea.org/newscenter/focus/fukushima/japan-re port.

[5] U. S. Nuclear Regulatory Commission, "Reactor Vessel Water Level Instrumentation in BWRs," Washington DC, 1984.

[6] Nuclear Regulatory Commission, Federal Register, Vol. 60, No. 175, 11 September 1995.

http://www.gpo.gov/fdsys/pkg/FR-1995-09-11/html/95-2 2461.htm

[7] D. D. Yue, "Use of PRA in Assessing BWR Vessel Level Instrumentation," Transactions of the American Nuclear Society, Vol. 50, 1985, pp. 358-359.

[8] U. S. Nuclear Regulatory Commission, "Resolution of Generic Safety Issues: Item B-60: Loose Parts Monitoring Systems (Rev. 1), NUREG-0933, Main Report with Supplements 1-33," 2012.

http://www.nrc.gov/reading-rm/doc-collections/nuregs/sta ff/sr0933/sec2/b60r1.html

[9] H. M. Hashemian, "Sensor Performance and Reliability," The Instrumentation, Systems, and Automation Society, 2005.

[10] S. Kiss, S. Lipcsei and J. Végh, "Overview of Recent KFKI AEKI Activities in the Field of Plant Surveillance and Diagnostics, Power Plant Surveillance and Diagnostics: Applied Research with Artificial Intelligence," Springer-Verlag, Berlin, Heidelberg, pp. 51-62

[11] J. Végh, et al., "Implementation of New Reactivity Measurement System and New Reactor Noise Analysis Equipment in a VVER-440 Nuclear Power Plant," IEEE Transactions on Nuclear Science, Vol. 57, No. 5, 2010, pp. 2689-2696.

[12] P. Jirsa, "Algorithm for Monitoring the Height of Flooding of the Core, Technical Note," Annals of Nuclear Energy, Vol. 24, No. 6, 1997, pp. 508-509.

[13] D. Wach and G. Kosály, "Investigation of the Joint Effect of Local and Global Driving Sources in Incore-Neutron Noise Measurements," Atomkernenergie, Vol. 23, 1974, pp. 244-250.

[14] T. Katona, L. Mesko, G. Por and J. Valko, "Some As- 
pects of the Theory of Neutron Noise," Progress in Nuclear Energy, Vol. 9, 1982, pp. 209-222. doi:10.1016/0149-1970(82)90044-0

[15] R. Kozma, "Studies on the Relationship between the Statistics of Void Fraction Fluctuations and Parameters of Two-Phase Flows," International Journal of Multiphase Flow, Vol. 21, No. 2, 1995, pp. 241-251. doi:10.1016/0301-9322(94)00077-W

[16] R. Kozma, H. van Dam and J. E. Hoogenboom, "Identification of Flow Patterns by Neutron Noise Analysis During Actual Coolant Boiling in Thin Rectangular Channels," Nuclear Technology, Vol. 100, 1992, pp. 97-110.

[17] R. Kozma, "Studies on the Relationship between the Statistics of Void Fraction Fluctuations and Parameters of Two-Phase Flows," International Journal of Multiphase Flow, Vol. 21, No. 2, 1995, pp. 241-251. doi:10.1016/0301-9322(94)00077-W

[18] R. Kozma, "Noise Investigations on Boiling Effects in a Simulated MTR-Type Fuel Assembly," Delft University Press, Delft, 1992.

[19] T. Katona and R. Kozma, "Problems of Estimation of the Thermohydraulic Parameters Using Neutron and Temperature Noise Signals," Progress in Nuclear Energy, Vol. 21, 1988, pp. 431-445. doi:10.1016/0149-1970(88)90061-3

[20] L. J. Kostić, J. Runkel and D. Stegemann, "Thermohydraulics Surveillance of Pressurized Water Reactors by Experimental and Theoretical Investigations of the Low Frequency Noise Field," Progress in Nuclear Energy, Vol. 21, 1988, pp. 421-430. doi:10.1016/0149-1970(88)90060-1

[21] T. H. J. J. van der Hagen and J. van der Voet, "Interpretation of Velocities Determined by Noise Analysis for Various Void Fractions and Flow Regimes in Two-Phase Flow," Progress in Nuclear Energy, Vol. 21, 1988, pp. 565-573. doi:10.1016/0149-1970(88)90077-7

[22] T. Katona and R. Kozma, "Studies to the Stochastic Theory of the Coupled Reactorkinetic-Thermohydraulic Systems: Analysis of Low-Frequency Noise Phenomena,": Central Rearch Institute for Physics, Budapest, 1986, 18 p.

[23] J. A. Thie, "Power Reactor Noise," American Nuclear Society, La Grange Park, 1981.

[24] R. Hampel, S. Fleischer, F. Dräger and T. Maekawa, "Water Level Measurement System For Boiling Water Reactors Using Internal Gamma Radiation-Neural Network Application," Proceedings of the 4th International FLINS Conference on Intelligent Techniques and Soft Computing in Nuclear Science and Engineering, Bruges, 28-30 August 2000, pp. 504-511.

[25] Core Exit Temperature (CET), "Effectiveness in Accident Management of Nuclear Power Reactor," Nuclear Energy Agency, Committee on the Safety of Nuclear Installations, 2010.

[26] T. Katona, "Investigation of the Possibility of Identification of Coolant Condition via Analysis of Local Temperature Noise (in Russian)," KFKI, Budapest, 1978, p. 23.

[27] R. Kozma and J. E. Hoogenboom, "Flow Measurements Using Noise Signals of Axially Displaced Thermocou- ples," Annals of Nuclear Energy, Vol. 17, No. 9, 1990, pp. 493-513. doi:10.1016/0306-4549(90)90004-W

[28] D. L. Nguyen, E. R. F. Winter and M. Greiner, "Sonic Velocity in Two-Phase Systems," International Journal of Multiphase Flow, Vol. 7, No. 3, 1981, pp. 311-320. doi:10.1016/0301-9322(81)90024-0

[29] J. L. Xu and T. H. Chen, "Acoustic Wave Prediction in Flowing Steam-Water Two-Phase Mixture," International Journal of Heat and Mass Transfer, Vol. 43, No. 7, 2000, pp. 1079-1088. doi:10.1016/S0017-9310(99)00213-6

[30] M. A. Grolmes and H. K. Fauske, "Propagation Characteristics of Compression and Rarefaction Pressure Pulses in One-Component Vapor-Liquid Mixtures," Nuclear Engeneering and Design, Vol. 11, No. 1, 1970, pp. 137 142. doi:10.1016/0029-5493(70)90024-5

[31] R. C. Mecredy and L. J. Hamilton, "The Effects of Nonequilibrium Heat, Mass and Momentum Transfer on Two-Phase Sound Speed," International Journal of Heat and Mass Transfer, Vol. 15, No. 1, 1972, pp. 61-72. doi:10.1016/0017-9310(72)90166-4

[32] L. I. Nikitin and N. V. Shariy, "On the Resonances of the Coolant in the Primary Circuit Loop of th VVERs," In: , A. A. Gusarov, Eds., Dinamical Streses and Deformations in Elements of Components of Energetics, Nauka, Moscow, 1977, pp. 67-76.

[33] J. A. Thie and J. A. Mullens, "Utilization of Pressure Noise for Reactor Anomaly Monitoring," Proceeding of Specialist's Meeting on Early Diagnosis of Failures in Primary System Components of Nuclear Power Plants, IAEA Report, Vienna, 1982.

[34] T. Katona and I. Nagy, "Interpretation of the Low-Frequency Pressure Fluctuations in PWR-s," 16th Informal Meeting on Reactor Noise, Budapest, 18-20 May 1983, pp. 9-17.

[35] I. Nagy and T. Katona, "Theoretical Investigation of the Low-Frequency Pressure Fluctuation in PWRs," Progress in Nuclear Energy, Vol. 15, No. 1-3, 1985, pp. 671-683. doi:10.1016/0149-1970(85)90096-4

[36] G. Grünwald, K. Junghanss and P. Liewers, "Investigation of Pressure Oscillations in a PWR Primary Circuit, Fourth Specialists Meeting on Reactor Noise Progress in Nuclear Energy, Vol. 15, 1985, pp. 651-659.

[37] G. Grünwald, G. Hessel, P. Liewers and W. Schmitt, "Low Frequency Pressure Oscillations in a PWR," Progress in Nuclear Energy, Vol. 9, 1982, pp. 569-579. doi:10.1016/0149-1970(82)90076-2

[38] V. V. Bulavin, D. F. Gutsev and V. J. Pavelko, "The Experimental Definition of the Acoustic Standing Waves Series, Formed in the Coolant of the Primary Circuit of VVER-440 Type Reactor," Progress in Nuclear Energy, Vol. 29, No. 3-4, 1995, pp. 153-170. doi:10.1016/0149-1970(95)00005-5

[39] E. Altstadt, et al., "Development of Theoretical Oscillation-model for WWER-type Reactors on the basis of Finite-element Method and Adjustment of Model using Measurement of Vibrations at Original Plant (in German)," Rossendorf, FZR-196, 1997.

[40] E. Altstadt, M. Scheffler and F.-P. Weiss, "Component 
Vibration of VVER-Reactors-Diagnostics and Modelling, Progress in Nuclear Energy, Vol. 29, No. 3-4, 1995, pp. 129-138. doi:10.1016/0149-1970(95)00002-2

[41] K. N. Proskuryakov, "Application of Vibroacoustic Noise for Diagnostics of Technological Process in Nuclear Power Plants," MEI, Moscow, 1999.

[42] G. Mondjtaba, "Numerical and Experimental Justification for Acoustic Models of Coolant in Components of NPPs at WWER and PWR," Ph.D. Thesis, 2004.

http://www.dissercat.com/content/raschetno-eksperimenta lnoe-obosnovanie-akusticheskikh-modelei-teplonositelya- v-oborudovanii

[43] K. S. Novikov, "Numerical and Experimental Justification of Reasons for Increasing Vibrations of in Vessel Components and Fuel Assemblies in WWER-1000 Type Reactors," Ph.D. Thesis, 2010.

http://www.dissercat.com/content/raschetno-teoretichesko e-i-eksperimentalnoe-obosnovanie-uslovii-rosta-vibratsiiv-vku-i-tvs

[44] T. Katona, "Statistical Analysis of Subcooled Boiling Acoustic Noise," KFKI, Budapest, 1981, p. 27. 